\title{
Imaging zero-offset 3D P-cable data with CRS method
}

\author{
M. Glöckner ${ }^{1}$, J. Walda ${ }^{1}$, S. Dell ${ }^{1}$, D. Gajewski ${ }^{1}$, J. Karstens ${ }^{2}$, D. Kläschen ${ }^{2}$, C. Berndt \\ ${ }^{1}$ University of Hamburg, Bundesstraße 55, D-20146 Hamburg (Germany).E-mail: martina.gloeckner@uni-hamburg.de \\ ${ }^{2}$ GEOMAR Helmholtz Centre, Kiel
}

\begin{abstract}
SUMMARY
Standard seismic acquisition and processing require appropriate source-receiver offsets. P-cable technology represents the opposite, namely, very short source-receiver offsets at the price of increased spatial and lateral resolution with a high-frequency source. To use this advantage, a processing flow excluding offset information is required. This aim can be achieved with a processing tuned to diffractions because point diffractions scatter the same information in offset and midpoint direction. Usually, diffractions are small amplitude events and a careful diffraction separation is required as a first step. We suggest the strategy to use a multiparameter stacking operator, e.g, common-reflection surface, and stack along the midpoint direction. The obtained kinematic wavefield attributes are used to calculate time-migration velocities. A diffractivity map serves as filter to refine the velocities. This strategy is applied to a 3D P-cable data set to obtain a time-migrated image.
\end{abstract}

Key words: Image processing, Wave scattering and diffraction

\section{INTRODUCTION}

The P-cable technology has been used for years. However, building a reliable velocity-model for 3D time and depth migration is still challenging. The P-cable technology consists of short streamers and a high frequency source. This leads to an increased spatial and lateral resolution with very small bin sizes. The short source-receiver offsets, however, have limitations to determine a velocity model with 


\section{M. Glöckner, J. Walda, S. Dell, D. Gajewski, J. Karstens, D. Kläschen, C. Berndt}

standard velocity analysis.

The need for a good and appropriate velocity model for time and depth migration is well-known, e.g., a residual move-out (RMO) analysis can be performed to obtain and update a velocity model (see for example Yilmaz (2001)). The method follows an iterative approach based on the analysis of flatness of events after migration. Therefore, the offsets for one specific midpoint are plotted and flatten by updating the velocity model. Another approach, frequently used in processing, is the common-offset migration and the application of inverse normal move-out correction followed by velocity analysis, (e.g. Bancroft et al. 1998). These two different methods of velocity-model building have in common, that they require appropriate, i.e., long offsets and a starting velocity model.

P-cable data do not allow model building by conventional methods if an insufficient offset-to-target ratio is present. Diffractions provide a tool for model building even for zero-offset (ZO) data (Bauer et al. 2017). In combination with the high-resolution potential of P-cable data, geological discontinuities, such as, faults, pinch-outs, and small-size scattering objects might be resolved (Klem-Musatov et al. 1994). These features are one of the objectives of seismic interpretation. The seismic response from these features is encoded in diffractions (Khaidukov et al. 2004). A separation of diffracted and reflected energy is mandatory to perform diffraction processing. First applications of diffraction imaging with P-cable data are shown by Merzlikin et al. (2017). They use azimuthal plane-wave destruction to detect faults and channels accounting for different orientations of edge diffractions. Klokov et al. (2017) expand this procedure and apply a 1D diffraction focusing analysis for time migration.

In this paper, we go a step further and perform a consistent velocity-model building based on kinematic wavefront attributes obtained by the common-reflection surface (CRS) method (Müller 1999). The first step is to separate reflections and diffractions. Furthermore, the diffraction-only data are stacked with a multiparameter stacking method. The byproduct of the stack are the kinematic wavefront attributes. These attributes are used to calculate a 3D velocity model and to perform, e.g., a time migration.

\section{METHOD}

This part describes the different steps to obtain a migrated time image. We start with the crucial step of separating diffractions from the total wavefield using wavefront attributes. Afterwards, we explain the model-building process and time migration. In all stages, we focus on diffractions because they are the key for the whole process to obtain a migrated image for P-cable data with their short source-receiver offsets. 


\subsection{Separation}

There are different possibilities to do the separation of diffractions from the data in time and depth domain. One approach, in depth, is to eliminate focused reflections and demigrate the residual wavefield to obtain diffractions (Moser \& Howar 2008). Mandatory for this approach is an good a prior depth-velocity model. Since we are working in the time domain, another possibility is to use kinematic wavefront attributes for separation, (e.g., Dell \& Gajewski 2011). A third option is to apply filters designed as finite-difference stencils for the plane-wave differential equation and decompose the seismic records into diffracted and reflected components using plane-waves, (e.g., Fomel 2002; Kozlov et al. 2004). Another method is presented by Schwarz \& Gajewski (2017). They calculate the dips and remove them between a user-defined threshold using adaptive subtraction.

We chose the plane-wave destruction method introduced by Claerbout et al. (1992) and Fomel (2002). They state that the seismic image is characterized by a superposition of local plane waves. Then, finite-difference filters are designed to destroy these waves. This procedure is done in two steps: first, estimate the dominant local slope by least-square optimization. Secondly, apply non-stationary planewave destruction filters (Fomel 2002). The remaining data consists the diffractions. This technique can be applied fast without further processing steps and is therefore, favored against the other techniques which require additional processing steps demanding offset information, e.g, the separation with kinematic wavefront attributes. Offset information are valuable for the separation process due to a more stable processing in terms of attribute determination which are used for the accordingly separation method. The separation without appropriate source-receiver offsets is a challenging task and must be performed with great care.

\subsection{Multiparameter Stack}

Conventionally stacking is performed along a hyperbola in offset direction. We face the issue that with P-cable data, offsets are very short and fitting hyperbolas is ambiguous. Therefore, we use a multiparameter stacking operator, which stacks the data in midpoint direction, too. There are several operators around, such as, CRS (Müller 1999; Bergler 2004), multifocusing (Landa et al. 2010), and non-hyperbolic CRS (Fomel \& Kazinnik 2013). Walda et al. (2017) claim that for complex media the choice of operator to determine wavefront attributes is not important since they provide very similar results. We use the so-called CRS operator to calculate the kinematic wavefront attributes

$t(\mathbf{m}, \mathbf{h})=\left(t_{0}+\mathbf{p}^{T} \mathbf{m}\right)^{2}+\frac{2 t_{0}}{v_{0}} \mathbf{m}^{T} \mathbf{R} \mathbf{K}_{N} \mathbf{R}^{T} \mathbf{m}+\frac{2 t_{0}}{v_{0}} \mathbf{h}^{T} \mathbf{R} \mathbf{K}_{N I P} \mathbf{R}^{T} \mathbf{h}$.

The coordinates are expressed in terms of midpoint $\mathbf{m}$, half-offset $\mathbf{h}$, and the zero-offset ( $\mathrm{ZO}$ ) travel time $t_{0}$. Bold capital letters indicate a $2 \times 2$ matrix, and bold small letters are $2 \mathrm{D}$ vectors, where the 
directions corresponds to inline and crossline, used in 3D processing. The three additional variables $\mathbf{p}$, $\mathbf{M}$ and $\mathbf{N}$ are the kinematic wavefront attributes (Hubral 1983). They describe the wavefront and ray geometry (Gelchinsky et al. 1999) and are defined by

$\mathbf{p}=\frac{-2 \sin \alpha}{v_{0}}\left(\begin{array}{c}\cos \beta \\ \sin \beta\end{array}\right), \quad \mathbf{M}=\mathbf{R} \mathbf{K}_{N I P} \mathbf{R}^{T}, \quad \mathbf{N}=\mathbf{R} \mathbf{K}_{N} \mathbf{R}^{T}$,

with

$\mathbf{R}=\boldsymbol{\Phi} \Theta, \quad \boldsymbol{\Phi}=\left(\begin{array}{cc}\cos \beta & -\sin \beta \\ \sin \beta & \cos \beta\end{array}\right), \quad \boldsymbol{\Theta}=\left(\begin{array}{cc}\cos \alpha & 0 \\ 0 & 1\end{array}\right)$.

Horizontal slowness $\mathbf{p}$ includes the dip angle $\alpha$ and azimuth angle $\beta$. The near-surface velocity $v_{0}$ is assumed to be known, which is particularly simple in our case since we use marine data in this work. The parameter $\mathbf{R}$ is a rotation matrix to the ray-centered coordinate system. The parameter $\mathbf{N}$ is a two-by-two curvature matrix containing the curvature of the normal $(\mathrm{N})$ wave $\mathbf{K}_{N}$ from a hypothetical wave starting from an exploding reflector segment and emerging at the surface. The parameter $\mathbf{M}$ is a two-by-two matrix containing the curvatures of the normal-incident point (NIP) wave $\mathbf{K}_{N I P}$ from a hypothetical wave starting from the NIP and emerging at the surface.

Considering ZO, as it is the case for our P-cable data, only two parameters have to be calculated: the slowness $\mathbf{p}$ and curvature matrix $\mathbf{N}$ (see 2). For a point diffractor $\mathbf{N}=\mathbf{M}$ is true. We favor the diffraction processing due to the fact that the same information in midpoint and offset direction are available for a point scatterer. In contrast to reflections, where the information are not the same and therefore cannot be used for the special P-cable case, where we deal with ZO data. In conclusion, the ZO CRS operator reduces to

$t(\mathbf{m}, \mathbf{h}=0)=\left(t_{0}+\mathbf{p}^{T} \mathbf{m}\right)^{2}+2 t_{0} \mathbf{m}^{T} \mathbf{R} \mathbf{K}_{N} \mathbf{R}^{T} \mathbf{m}$.

\subsection{Time Migration}

Normally, offsets are crucial to derive a migration velocity-model. In the absence of this information, we use the wavefront attributes of diffractions to determine a velocity model, which can be used for time migration. Spinner (2006) present a method to calculate the velocity model from the kinematic wavefront attributes using CRS method. She expresses the diffraction CRS operator in apex coordinates and relates the kinematic wavefront attributes with the time-migration velocity

$\mathbf{V}^{2}=\left(\mathbf{p}^{T} \mathbf{p}+\frac{2 t_{0}}{v_{0}} \mathbf{N}\right)^{-1}$

To further improve the resulting velocity model, we use the approach discussed in Glöckner et al. (2019a). They used a migrated coherence section to weight the velocity model, interpolate gaps and smooth the velocity model. We adopt this approach for the azimuth section and calculate coherence 
in terms of semblance Neidell \& Taner (1971) to determine regions with diffractions. This section is weighted with the velocity model to enhance appropriately determined velocities for diffractions. Afterwards, we apply interpolation and smoothing.

The diffraction processing is well suited for a following 3D Kirchhoff time migration, which can be seen as a diffraction operator and is similar to our approach to use diffractions for the P-cable data processing. Because of short P-cable offsets, we can only perform a 3D post stack Kirchhoff time migration.

The next section presents the application of our proposed method to a 3D p-cable data set.

\section{APPLICATION}

\subsection{Data}

We have applied this method to a P-cable cube that was recorded by the German research vessel 'Sonne' in late 2016 (Berndt et al. 2016). The cruise investigated the sector collapse of the island Ritter that took place in 1888 to constrain the slide parameters and the tsunami potential of such events. Volcanic island flank collapses have the potential to trigger devastating tsunamis threatening coastal communities and infrastructure (Karstens et al. 2019). Understanding this process is crucial for assessing the hazard potential of volcanoes with slowly deforming flanks like, e.g., Mt Etna and Kilauea. Ritter Island is a volcanic island located $100 \mathrm{~km}$ northeast of New Guinea in the centre of the Bismarck volcanic arc. The present-day morphology is dominated by a horse-shoe shaped scarp formed by the 1888 flank collapse. The uninhabited island is $2 \mathrm{~km}$ long, $200 \mathrm{~m}$ wide and $140 \mathrm{~m}$ high with steep flanks. Prior to the collapse, the island was about $800 \mathrm{~m}$ high and had a diameter of $2 \mathrm{~km}$. German colonists documented the generated tsunami, caused by the largest historic flank collapse, and measured run-up heights of $20 \mathrm{~m}$, arrival times, and report damages. These reports are valuable and unique for the estimation of the tsunami potential.

The seismic data are recorded with the 3D P-cable seismic system manufactured by Geometrics. In total sixteen digital streamers were used, four are solid state and twelve are oil-filled. Each streamer had eight hydrophone groups spaced $1.5 \mathrm{~m}$ apart and the offset between source and receivers varies between $160 \mathrm{~m}$ and $320 \mathrm{~m}$. The source consisted of two GI guns taht were triggered with a shot interval of approximately $10 \mathrm{~m}$ (Berndt et al. 2016). The data were pre-processed including the following steps: re-positioning of streamers, 3D binning, frequency filtering $(45-240 \mathrm{~Hz})$. Additional processing steps are: a normal move-out correction with water velocity, and stacking. 


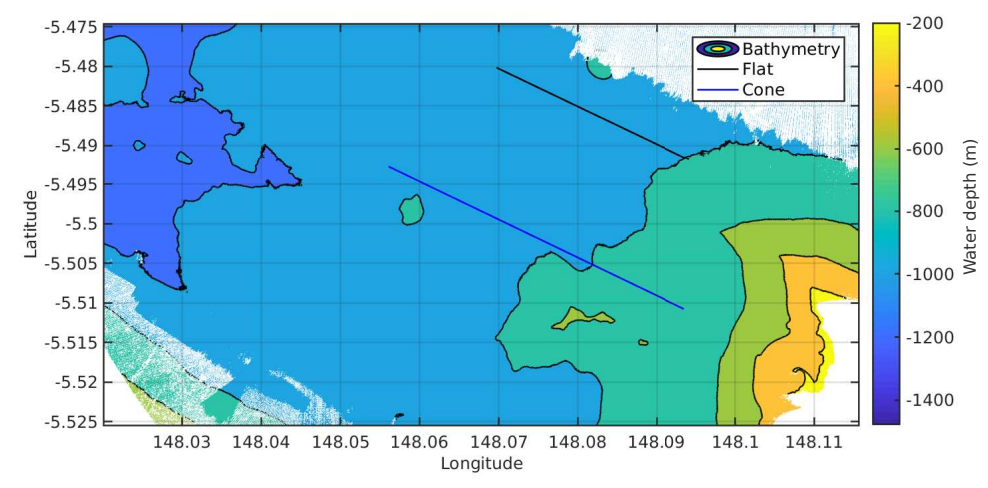

Figure 1. Bathymetry of the P-cable data under investigation. Ritter island is located in the Northeast. The two lines show the example crosslines.

\subsection{Results}

We illustrate and discuss the different steps of our proposed method exemplary for a part of the acquisition shown in Figure 1. This part shows the proximal area of the land slide. The slope of Ritter island and smaller submarine volcanic cones are visible.

We use two different parts of the cube, marked in Figure 1, namely a section with a volcanic cone that predates the sector collapse and little sedimentary layering (cone area, blue line), and a section with more sedimentary layering and no cones (flat area, black line). These areas are exemplary used in the further description and visualization of our method, in addition with 3D views of the full data set.

The first step in our work flow is the event separation. Only the assumed point diffractions yield information in midpoint direction, which are necessary to perform a stack and calculate a migrationvelocity model. Therefore, a suitable separation is a crucial step to obtain the needed diffraction-only data. Figure 2 shows the original data (a) and the separated data after plane- wave destruction (b) for the cone area. Diffractions are better visible after the separation. In addition, reflections in the dipping flank and internal reflections in the middle are reduced. Nevertheless, some artifacts remain, especially for dipping events close to the cone, which should not be considered as pure point diffractions.

Figure 3 shows the original data (a) and separated data (b) for the flat area.Here, the PWD worked better when compared to the cone area, because more sedimentary layering is removed and the diffractions are better visible. Reflections at $1.4 \mathrm{~s}$ are well attenuated and show the diffraction energy in the data.

The next step is to perform the multiparameter stacking with the diffraction-only data to obtain the stack and coherence sections, as well as, the mentioned kinematic wavefront attributes. Due to the preprocessing offset information are not available, we perform the stack along the midpoint direction. 


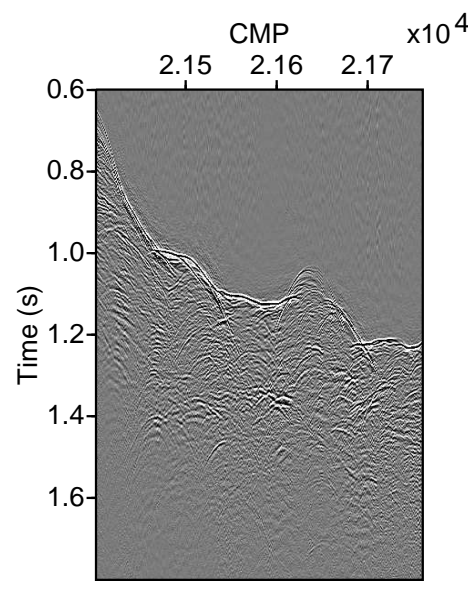

(a) Original data for cone area.

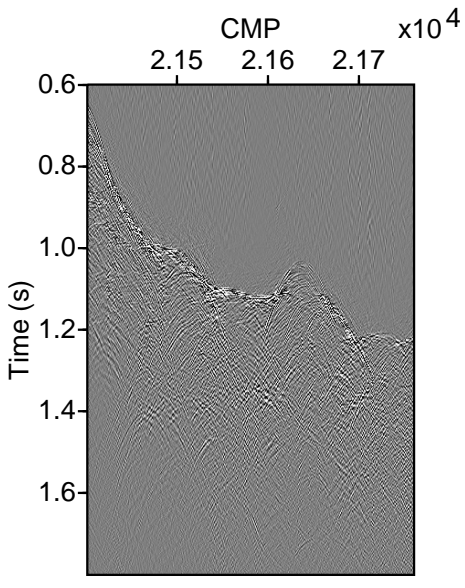

(b) Separated data for cone area.

Figure 2. Comparison of original and separated data for the area with volcanic cones.

Figure 4 shows the stacked sections of the separated diffraction data. The multiparameter stack leads to an improved signal-to-noise ratio and diffractions are better recognizable. Both sections show clear diffraction events in absence of reflections which mask the richness of diffracted wavefield due to higher amplitudes.

Figure 5 shows a 3D view of the processed P-cable volume. On the right side (east), we see the layered flank of the island. In the middle of the image is a volcanic cone. There, we observe some internal events. The time slice shows circular shaped events which indicate point diffractions. Furthermore, we see that the stack enhances the signal-to-noise ratio in comparison to Figures 2 and 3.

Another important attribute, the coherence, is shown in Figure 6. The cone area (a) as well as the flat area (b) show the diffractions with higher coherence values than the background. On-sided diffraction tails are more visible than full diffraction hyperbolas.

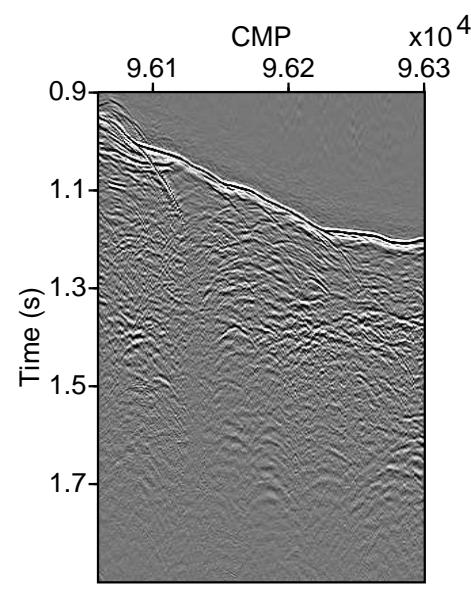

(a) Original data for flat area.

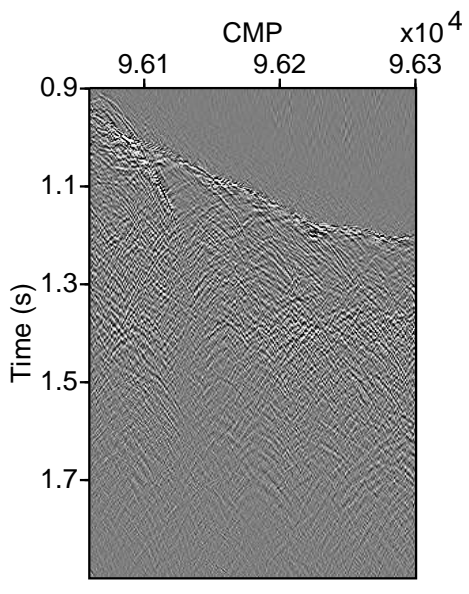

(b) Separated data for flat area.

Figure 3. Comparison of original and separated data for the area with flat sediments. 


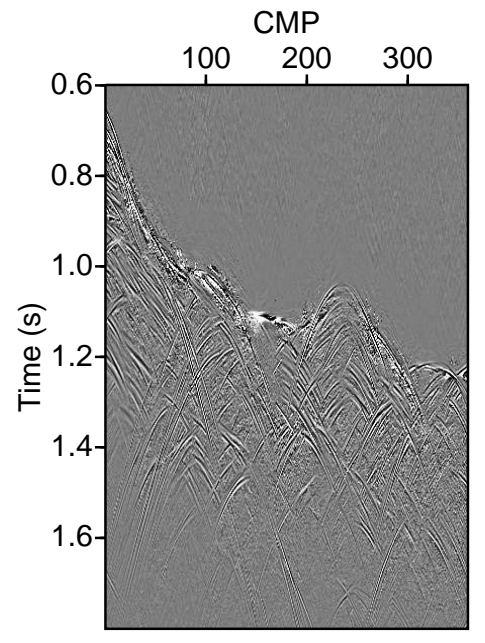

(a) Diffraction stack for cone area.

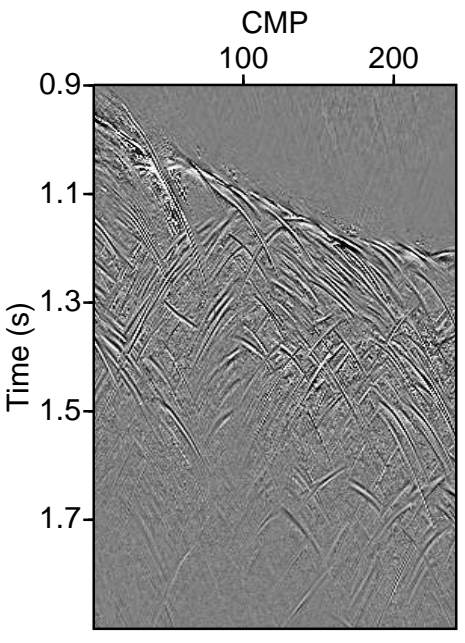

(b) Diffraction stack for flat area.

Figure 4. Diffraction stacks for the different example areas showing the diffraction potential of the data.

Furthermore, we obtain the kinematic wavefront attributes: the slowness vector and the curvature matrix of the diffracted wave. The attributes are divided in inline and crossline direction. Inline attributes are weaker in terms of quality in comparison to the corresponding crossline attribute due to the special acquisition geometry of P-cable data. We weight our attribute sections with the coherence to simplify the interpretation of the different attribute sections. Therefore, we define a threshold (here 0.1 ) in the coherence section and use it as weight for the attributes. That means, values of the coherence section above the threshold are multiplied with one, and values below wit zero, respectively. The slowness is divided in dip and azimuth (see Figures 7 and 8).

Figure 7 shows the dip section for the areas under investigation. Dip ranges from -27 to 27 degrees,

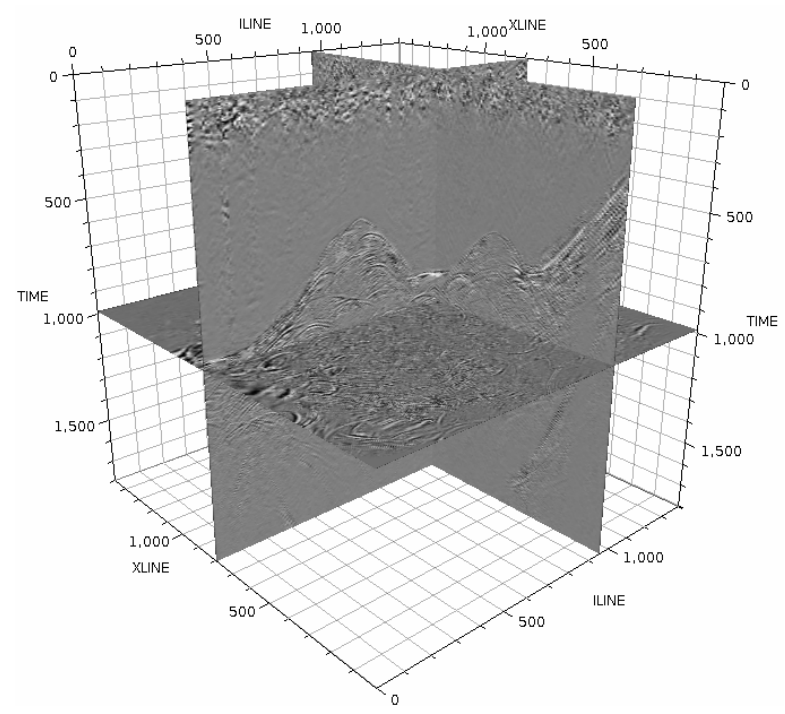

Figure 5. Diffraction stack of the processed P-cable data. The viewing direction is from south to north. 


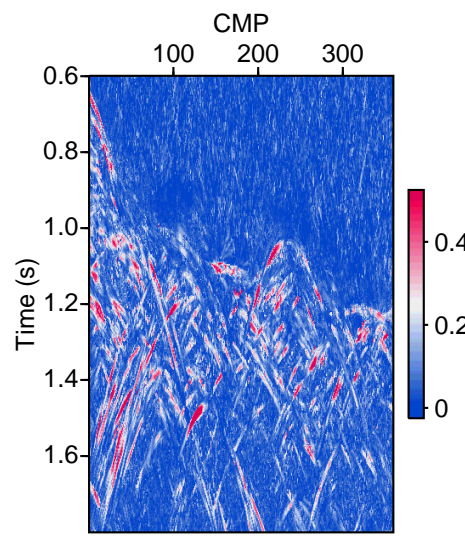

(a) Coherence for cone area.

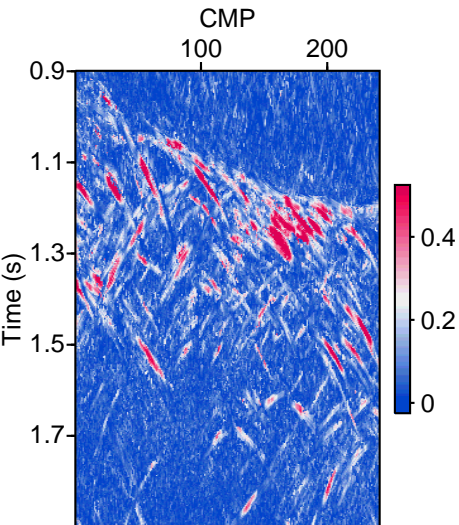

(b) Coherence for flat area.

Figure 6. Coherence section for the different example areas.

which is normal when considering diffractions with steep dipping tails. Events are also visible on the basis of dip information. The dip attribute can be used for a quality control of the separation. Here, only few events with dips close to zero are visible. Small dips are usually attributed to indicating reflections. However. the apex region of the diffractions have also dips close to zero, which is better visible in the flat area (b).

Figure 8 shows the azimuth section for the areas under investigation. We obtain narrow azimuths due to the acquisition geometry and the special P-cable technology. The azimuth images for the cone area (a) as well as the flat area (b) show many diffraction events.

In addition, we obtain the curvature matrix of the diffracted wave, which represents the second order derivatives of travel times in inline and crossline direction, and consists of three independent entries (see Figures 9, and 10). Figure 9 shows the different curvature matrix entries for the cone area and represent inverse curvature radii. The images are not as clean as the dip and azimuth images, due to their second-order derivative nature. Especially image (a) and (b) show diffractions, which have high

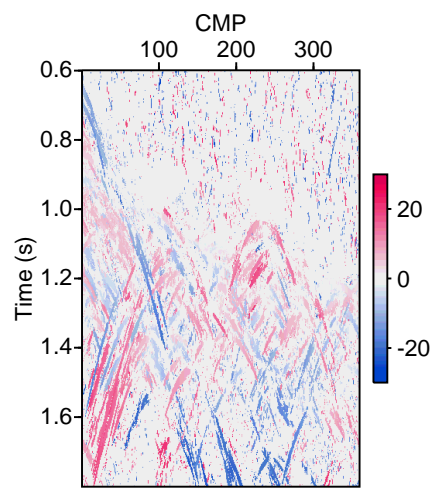

(a) Dip for cone area.

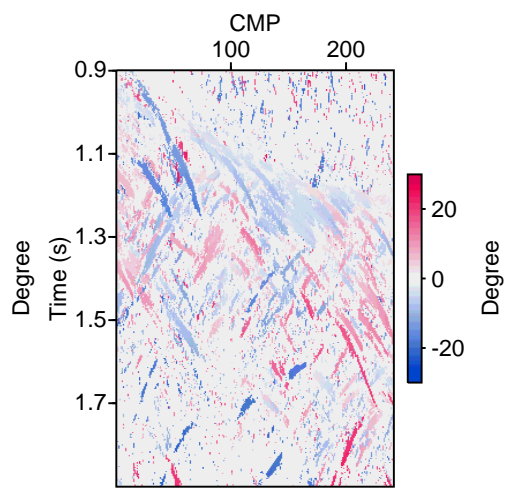

(b) Dip for flat area.

Figure 7. Dip angle for the different example areas. 


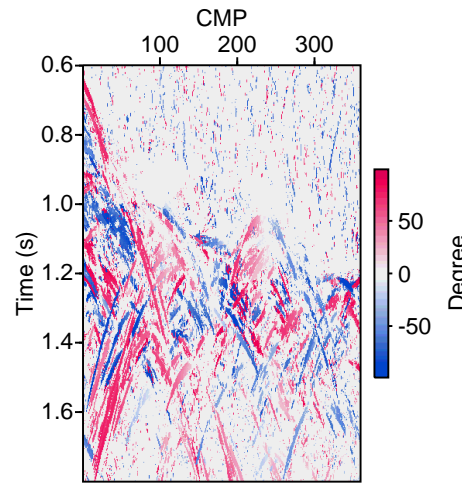

(a) Azimuth for cone area.

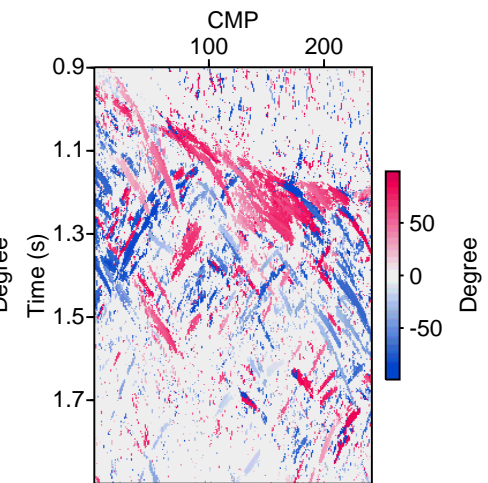

(b) Azimuth for flat area.

Figure 8. Azimuth for the different example areas.

curvatures. The values close to the sea bottom are more clean than deeper parts. The attribute $N_{01}$ from the mixed second-order derivative is the most unreliable and noisiest one.

Figure 10 shows the different curvatures for the area dominated by sedimentary layering. Here, events are better visible in comparison to Figure 9. Again, attributes $N_{00}$ (a) and $N_{11}$ (c) show clearer events in comparison with $N_{01}$ (b).

Now, all attributes needed for velocity-model calculation are available and time migration can be performed. Figure 11 shows the calculated time-migration velocities for both example areas. In general, velocities show appropriate values but some artifacts are visible. Higher velocities (in red) occur close to the ocean bottom for the flat area b) and on the left part for the cone area a). A reason for this is the difficult separation process and the stacking in just the common-midpoint (CMP) direction. The attributes, needed for the calculation of the velocities, are in some areas noisy and lead to erroneous velocities. Especially, reflection artifacts from the ocean bottom increase velocities due to insufficient curvature radii.

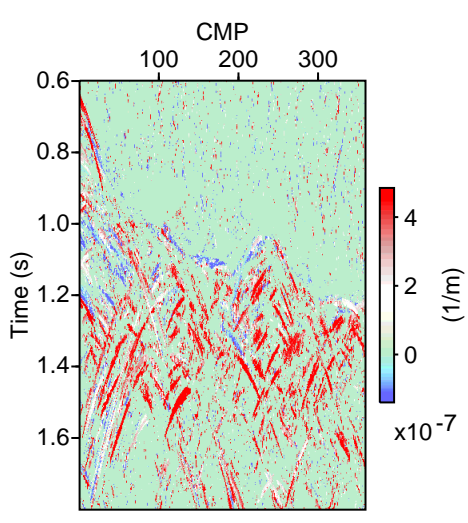

(a) $N_{00}$ for cone area

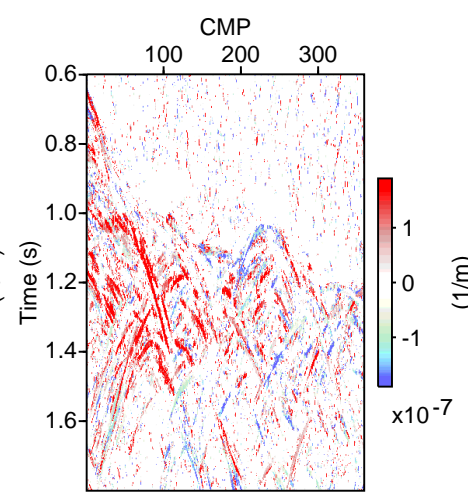

(b) $N_{01}$ for cone area.

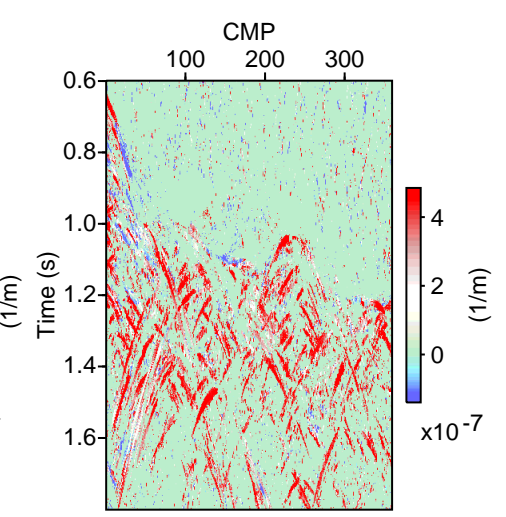

(c) $N_{11}$ for cone area.

Figure 9. Curvatures of diffraction-only data for the cone area. 


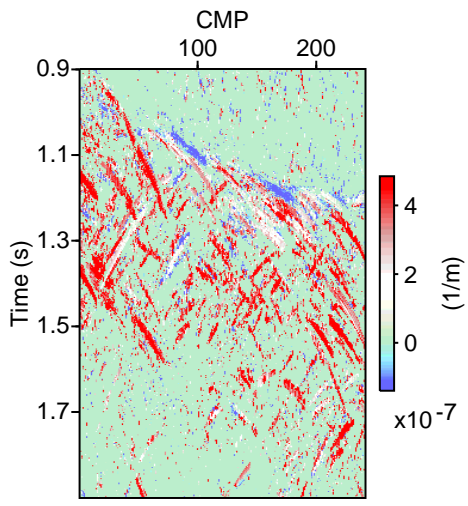

(a) $N_{00}$ for flat area.

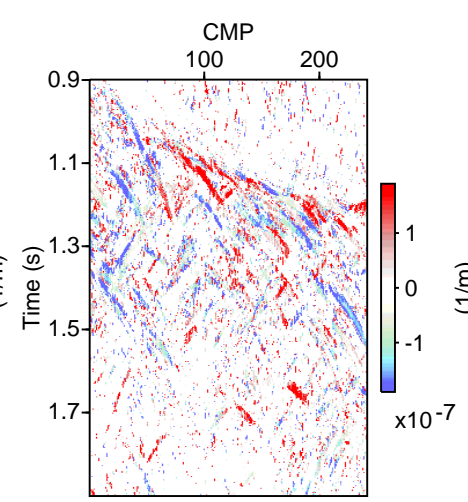

(b) $N_{01}$ for flat area.

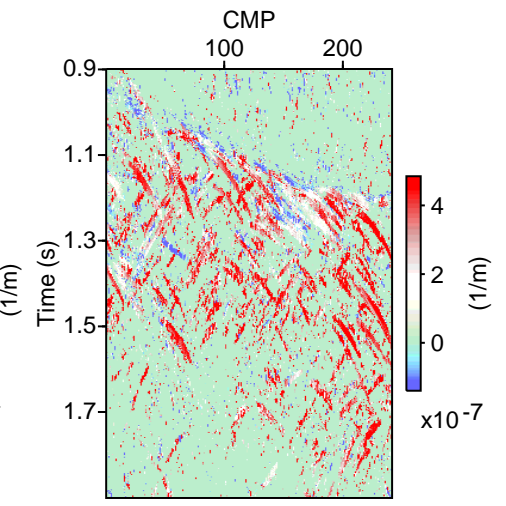

(c) $N_{11}$ for flat area.

Figure 10. Curvatures of diffraction-only data for area with sedimentary layering.

The migrated images are shown in Figure 12. The sea bottom is visible with the typical time-migration artifacts, which are mainly covered by the mute of the water column. The cone in Figure 12 (a) is less prominent than in Figure 2 or Figure 4 (a). However, we observe further events between 1.2 and 1.4 $\mathrm{s}$. The migrated image for the flat area (b) shows a structure in the middle between 1.1 and $1.3 \mathrm{~s}$. The velocity is less reliable in this area (see Figure $11 \mathrm{~b}$ ) due to high velocity spots in the upper part. Figure 13 shows a 3D-view of the migrated P-cable volume and is the same as for Figure 5 for comparison. The cone in the migrated image is smaller than in the stacked image. Furthermore, we see sedimentary layering under the ocean bottom. The time slice shows a complex pattern in the south-west related to the diffractions there.

The application shows that the proposed approach leads to a time-migrated image. We are able to produce this without any offset information using a work flow designed for diffractions.

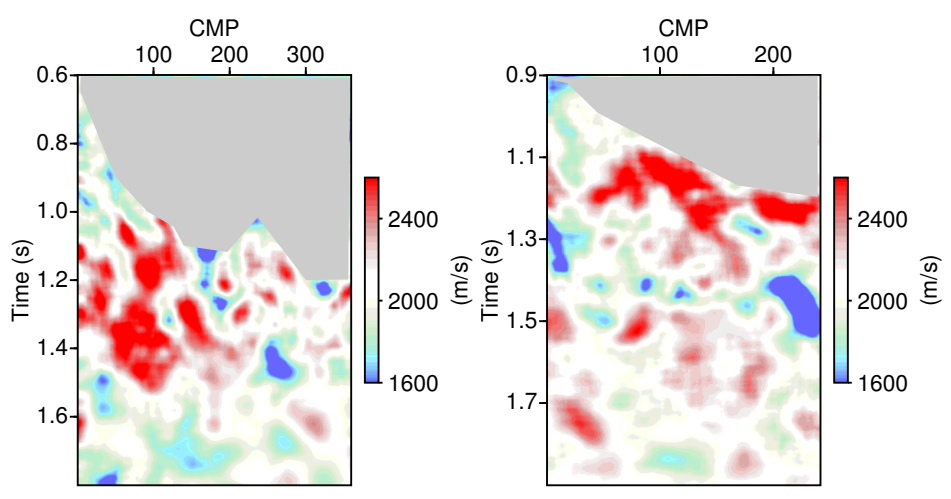

(a) Time-migration velocity for cone (b) Time-migration velocity for flat area. area.

Figure 11. Time-migration velocities for the different example areas. A mute of the water column (gray) is applied. 


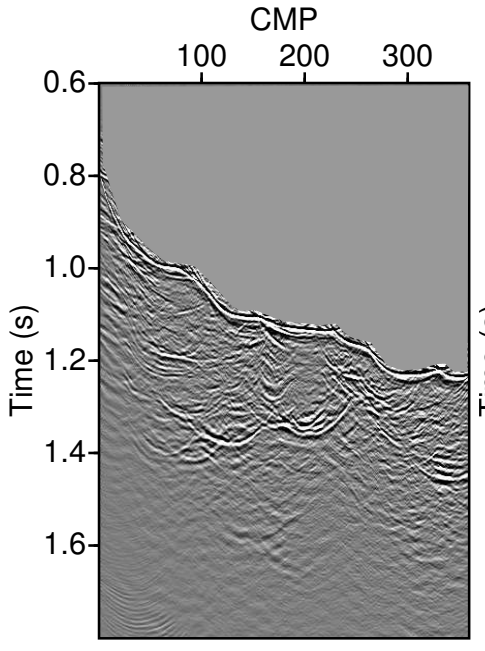

(a) Migrated image for cone area.

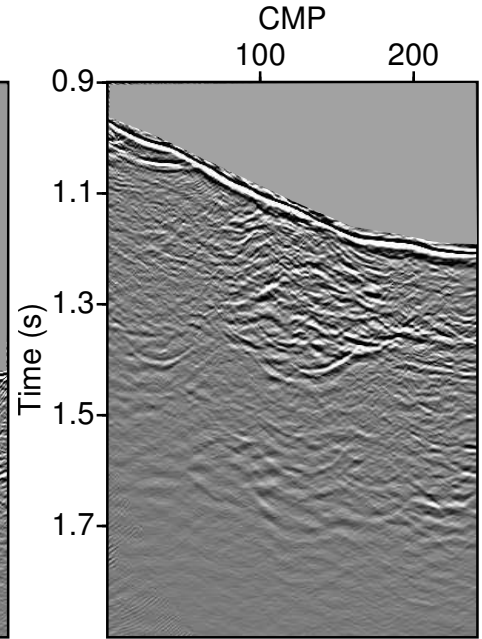

(b) Migrated image for flat area.

Figure 12. Time migration results for both example areas. The water column is muted.

\section{DISCUSSION}

In this section, we discuss the advantages, challenges, and issues of the proposed approach to obtain a velocity model and perform a time migration for 3D P-cable data. The short source-receiver offsets from P-cable data make standard velocity analysis almost impossible. The CRS-based diffraction processing with kinematic wavefront attributes for such data sets allows for the determination of a velocity model suitable for time migration. Unfortunately, different preprocessing steps, e.g. interpolation and filter methods, reduce the diffraction energy because they limit or smear over dip ranges. Preprocessing must be performed diffraction-friendly to preserve especially large dip values, which

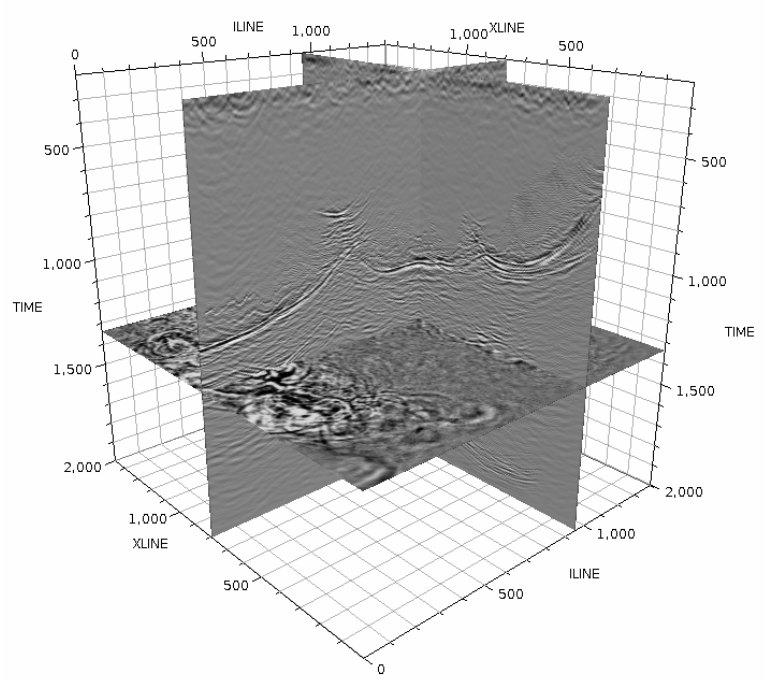

Figure 13. Time-migrated image of the processed P-cable data. The viewing direction is from south to north. 
are typical for diffractions.

The assumption that we handle with pure point diffractions offers the possibility to use a multiparameter stacking operator in midpoint direction instead of offset. Because of the weak amplitudes of diffractions, we have to perform a careful diffraction separation. Here, we are using the plane-wave destruction introduced by Fomel (2002). The separation is the crucial point in this approach and has room for improvements as shown in Merzlikin et al. (2017). They apply the plane-wave destruction for different azimuths and achieve improved results. We are not able to apply this procedure due to limited azimuths in our data set.

The time-migration velocities obtained from the kinematic wavefront attribute can be further improved. We must not forget, that for velocity analysis appropriate offset information are crucial. For the P-cable data sets, we are missing this kind of information. Therefore, we just applied a smoothing of time-migration velocities due to a needed smooth velocity distribution for time migration.

The time-migrated images show that our method generally works. We propose a consistent way to achieve a migrated image without additional information, such as ocean-bottom seismometer or long offset 2D surveys.

First attempts to perform velocity-model building for depth migration are done by Glöckner et al. (2018) and Glöckner et al. (2019b). They use the kinematic wavefront attributes as input for the wavefront tomography (Duvenick 2004; Klüver 2007). The wavefront tomography consists of two parts: the picker and the inversion. The automatic picker chooses samples with a user-defined coherence value and the corresponding kinematic wavefront attributes. These values are used for the inversion to calculate a depth-velocity model. Glöckner et al. (2019b) show that it is crucial to eliminate as much non-point-diffraction energy as possible. Reflection artifacts boost velocities and lead to overestimated velocity models which are not yet suitable for a depth migration. Furthermore, the second-order curvature attributes are less reliable then the first-order slowness attributes. They are more stable, when having appropriate source-receiver offset for the calculation. Bauer et al. (2019) show an approach for the wavefront tomography without the critical second-order curvatures. They use a diffraction focusing criterion for the inversion process. The separation is the most important part for a successful application. This issue must be addressed in future research to obtain improved velocity models for time and depth migration.

\section{CONCLUSIONS}

The advantage of P-cable technology is the increased spatial and lateral resolution when compared to conventional 3D data. The short source-receiver offsets prevent standard velocity analysis. We suggest an approach, which can fill this gap, focusing on the diffracted wavefield using the CRS operator. 


\section{M. Glöckner, J. Walda, S. Dell, D. Gajewski, J. Karstens, D. Kläschen, C. Berndt}

We first perform the separation of diffractions from the total wavefield using plane-wave destruction followed by a multiparameter stack on the diffraction data. Further outputs of the stacking are the kinematic wavefront attributes. These attributes allows us to calculate a velocity model for time migration. The proposed method is applied to a 3D P-cable data set from the Bismarck Sea. The data application shows the challenges when processing data without offset information. Diffractions are the tool to process such data sets which have the potential for time and depth velocity-model building.

\section{ACKNOWLEDGMENTS}

We thank the members of the Applied Seismic Group Hamburg for continuous discussions. This work was partly supported by the sponsors of the Wave Inversion Technology (WIT) Consortium. The data were collected with BMBF funding through the Ritter Island project ( 03G0252A ). We appreciate the constructive criticism of an anonymous reviewer and Alexey Stovas that helped to improve the paper.

\section{REFERENCES}

Bancroft, J. C., Geiger, H. D., \& Margrave, G. F., 1998. The equivalent offset method of prestack time migration, Geophysics, 63(6), 2042-2053.

Bauer, A., Schwarz, B., \& Gajewski, D., 2017. Utilizing diffractions in wavefront tomography, Geophysics, 82(2), R65-R73.

Bauer, A., Diekmann, L., \& Gajewski, D., 2019. Wavefront Tomography with Enforced Diffraction Focusing, in Expanded Abstracts, EAGE.

Bergler, S., 2004. On the determination and use of kinematic wavefield attributes for 3D seismic imaging, Ph.D. thesis, University of Karlsruhe.

Berndt, C., Muff, S., Klaucke, I., Watt, S., Böttner, C., Schramm, B., Völsch, A.-M., Bennecke, S., Elger, J., Chi, W.-C., Van Haren, J., Micallef, A., \& Roth, T., 2016. RV SONNE 252 Cruise Report: Tsunami potential of volcanic flank collapses, Tech. rep.

Claerbout, J. F., Karrenbach, M., \& Balog, O., 1992. Earth Soundings Analysis: Processing versus Inversion, Blackwell Sci. Publ. Inc.

Dell, S. \& Gajewski, D., 2011. Common-reflection-surface-based workflow for diffraction imaging, Geophysics, 76, S187-S195.

Duvenick, E., 2004. Tomographic determination of seismic velocity models with kinematic wavefield attributes, Ph.D. thesis, University of Karlsruhe.

Fomel, S., 2002. Applications of plane-wave destruction filters, Geophysics, 67(6), S139-S147.

Fomel, S. \& Kazinnik, R., 2013. Non-hyperbolic common reflection surface stack, Geophysical Prospecting, 61, 21-27. 
Gelchinsky, B., Berkovitch, A., \& Keydar, S., 1999. Multifocusing homeomorphic imaging Part 1. Basic concepts and formulas, Journal of Applied Geophysics, 42, 229-242.

Glöckner, M., Gajewski, D., Kläschen, D., Muff, S., \& Berndt, C., 2018. Wavefront tomography with difraction-only 3D P-cable data, in Expanded Abstracts, Soc. of Expl. Geophys.

Glöckner, M., Dell, S., Schwarz, B., Vanell, C., \& Gajewski, D., 2019a. Automated velocity model building and migration deconvolution in the time domain, Geophysics, 84, S229-S238.

Glöckner, M., Walda, J., Gajewski, D., Roth, T., \& Berndt, C., 2019b. Challenges in velocity-model building with 3D P-cable data, in Expanded Abstracts, EAGE.

Hubral, P., 1983. Computing true amplitude reflections in a laterally inhomogeneous earth, Geophysics, 48, 1051-1062.

Karstens, J., Berndt, C., Urlaub, M., Watt, S. F., Micallef, A., Ray, M., Day, S. J., Klaucke, I., Kühn, M., Muff, S., Klaeschen, D., Böttner, C., Schramm, B., Elger, J., Roth, T., \& Brune, S., 2019. From gradual spreading to catastrophic collapse - 3D seismic reconstruction of the 1888 Ritter Island volcanic sector collapse, Journal of Earth and Planetary Science, 517, 1-13.

Khaidukov, V., Landa, E., \& Moser, T. J., 2004. Diffraction imaging by focusing-defocusing: An outlook on seismic superresolution, Geophysics, 69(10), 1478-1490.

Klem-Musatov, K., Hron, F., \& Lines, L., 1994. Theory of Seismic Diffractions, Society of Exploration Geophysicists.

Klokov, A., Trevino, R. H., \& Meckel, T. A., 2017. Diffraction imaging for seal evaluation using ultra high resolution 3D seismic data, Marine and Petroleum Geology, 82, 85-96.

Klüver, T., 2007. Velocity model building using analytic and model-based diffraction traveltime functions, Ph.D. thesis, University of Karlsruhe.

Kozlov, E., Barasky, N., Korolev, E., Antonenko, A. P., \& Koshchuk, E., 2004. Imaging scattering objects masked by specular reflections, in Expanded Abstracts, Soc. of Expl. Geophys.

Landa, E., Keydar, S., \& Moser, T. J., 2010. Multifocusing revisited - inhomogeneous media and curved interfaces, Geophysical Prospecting, 58, 925-938.

Merzlikin, D., Meckel, T. A., Fomel, S., \& Sripanich, Y., 2017. Diffraction imaging of high-resolution 3D P-cable data from the Gulf of Mexico using azimuthal plane-wave destruction, FirstBreak, 35, 35-41.

Moser, T. \& Howar, C., 2008. Diffraction imaging in depth, Geophysical Prospecting, 56, 627-641.

Müller, T., 1999. Seismic imaging without explicit knowledge of the velocity model, Ph.D. thesis, University of Karlsruhe.

Neidell, N. \& Taner, T. M., 1971. Semblance and other coherency measures for multichannel data, Geophysics, 36, 482-497.

Schwarz, B. \& Gajewski, D., 2017. Accesing the diffracted wavefield by coherent subtraction, Geophysical Journal International, 211, 45-49.

Spinner, M., 2006. 3D CRS-based limited aperture Kirchhoff time migration, in Expanded Abstracts, Soc. of Expl. Geophys. 
16 M. Glöckner, J. Walda, S. Dell, D. Gajewski, J. Karstens, D. Kläschen, C. Berndt

Walda, J., Schwarz, B., \& Gajewski, D., 2017. A competitive comparison of multiparameter stacking operators, Geophysics, 82(4), V275-V283.

Yilmaz, O., 2001. Seismic Data Analysis, SEG, Tulsa. 